\title{
Tentativa de suicídio em residentes de São Paulo: aspectos epidemiológicos
}

\author{
Suicide attempt in residents of São Paulo: epidemiological aspects \\ Intento de suicidio en los residentes de São Paulo: aspectos epidemiológicos
}

\begin{abstract}
RESUMO
Objetivo: analisar o perfil epidemiológico das tentativas de suicídio na população residente da cidade de São Paulo durante o ano de 2015. Método: Trata-se de um estudo transversal, descritivo. O período de coletas ocorreu entre os meses de janeiro a dezembro de 2015, a base de dado elegível foi o sítio eletrônico do Departamento de Informática do Sistema Único de Saúde (Datasus), para análise das notificações registradas no SIVVA/Sinan, foram calculadas medidas de frequências das variáveis. Resultado: Foram identificadas 910 tentativas de suicídio sendo 44, 5\% no sexo feminino e 55,5\% no sexo masculino. Acerca da faixa etária 3,2\% eram crianças ( 0 a 9 anos de idade); 22,3\% adolescentes (10 a 19 anos de idade); $72,1 \%$ adultos ( 20 a 59 anos de idade) e 2,4\% idosos (60 anos e mais de idade). No que tange a variável raça/cor; branca (36,7\%), preto/pardo $(37,9 \%)$, indígena $(0,4 \%)$, amarela $(0,3 \%)$ e os demais dados foram ignorados. Conclusão: o estudo evidenciou que as tentativas de suicídio ocorrem principalmente entre a população adulta, ressalta-se a necessidade de reforçar quanto aos serviços de saúde estarem sensiveis ao seu registro atentando quanto aos cuidados a serem promovidos, mediante sua magnitude.
\end{abstract}

DESCRITORES: Tentativa de suicídio; Saúde mental; Serviços de Saúde Mental; Assistência á Saúde Mental.

\section{ABSTRACT}

Objective: to analyze the epidemiological profile of suicide attempts in the population living in the city of São Paulo during 2015. Method: This is a cross-sectional, descriptive study. The collection period occurred between January and December 2015, the eligible data base was the website of the Department of Informatics of the Unified Health System (Datasus), for analysis of the notifications recorded in the SIVVA/Sinan, and frequency measurements of the variables were calculated. Result: 910 suicide attempts were identified, $44,5 \%$ female and $55.5 \%$ male. About the age group $3.2 \%$ were children ( 0 to 9 years old); $22.3 \%$ adolescents ( 10 to 19 years of age); $72.1 \%$ adults ( 20 to 59 years of age) and $2.4 \%$ elderly (60 years and older). With regard to the variable race/color; white $(36.7 \%)$, black/brown $(37.9 \%)$, indigenous $(0.4 \%)$, yellow $(0.3 \%)$ and the other data were ignored. Conclusion: the study showed that suicide attempts occur mainly among the adult population, emphasizing the need to reinforce the need for health services to be sensitive to their record regarding the care to be promoted, due to their magnitude.

DESCRIPTORS: Suicide attempt; Mental health; Mental Health Services; Mental Health Care.

\section{RESUMEN}

Objetivo: analizar el perfil epidemiológico de los intentos de suicidio en la población que vive en la ciudad de Sao Paulo durante 2015. Método: Este es un estudio transversal y descriptivo. El período de recolección se produjo entre enero y diciembre de 2015, la base de datos elegible fue el sitio web del Departamento de Informática del Sistema Unificado de Salud (Datasus), para el análisis de las notificaciones registradas en el SIVVA/Sinan, y se calcularon las mediciones de frecuencia de las variables. Resultado: se identificaron 910 intentos de suicidio, 44,5\% mujeres y 55,5\% hombres. Alrededor del grupo de edad 3,2\% eran niños $(0$ a 9 años de edad); $22,3 \%$ adolescentes ( 10 a 19 años de edad); $72,1 \%$ adultos ( 20 a 59 años de edad) y 2,4\% de edad avanzada (60 años 0 más). Con respecto a la raza/color variable; blanco (36,7\%), negro/marrón (37,9\%), indígena (0,4\%), amarillo $(0,3 \%)$ y los otros datos fueron ignorados. Conclusión: el estudio mostró que los intentos de suicidio ocurren principalmente entre la población adulta, haciendo hincapié en la necesidad de reforzar la necesidad de que los servicios de salud sean sensibles a su historial con respecto a la atención a promover, debido a su magnitud.

DESCRIPTORES: Intento de suicidio; Salud mental; Servicios de Salud Mental; Atención de salud mental.

RECEBIDO EM: 16/11/2020 APROVADO EM: 16/11/2020 


\section{Leticia Corradi}

Acadêmica do Curso de Medicina da Universidade Santo Amaro- São Paulo- SP.

ORCID: 0000-0003-4822-4217

\section{Cintia Leci Rodrigues}

Biomédica, Mestre em Saúde Pública da Faculdade de Saúde Pública- USP, Docente do Curso de Medicina da Universidade Santo Amaro. São Paulo- SP.

ORCID: 0000-0001-8064-2203

\section{Jefferson Carlos de Oliveira}

Enfermeiro, Mestrado Profissional em Enfermagem- Enfermagem no Processo de Cuidar em Saúde do Centro Universitário São Camilo, Docente do Curso de Enfermagem e Pós-Graduação em Urgência e Emergência- Centro Universitário Anhanguera de São Paulo- Vila Mariana.

ORCID: 0000-0002-5258-7099

\section{INTRODUÇÃO}

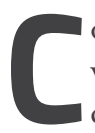

onceituado como lesão autoprovocada e incluído nas categorias que vão de X60 a X84 da Classificação Estatística Internacional de Doenças e Problemas Relacionados à Saúde (conhecida pela sigla CID) 10a Revisão ${ }^{1}$, segundo Tavares1, o suicídio é um fenômeno vivenciado por pessoas que compreendem, na realização desse ato, a libertação de uma dor psicológica intolerável ${ }^{1}$.

O suicídio deriva da influência de mútuos fatores podendo ser de ordem, antropológica, psicológica, filosófica, biológica e social tendo sua consumação derivada de ato incitado e realizado pelo indivíduo com o objetivo de pôr fim à vida $^{2}$. Contudo, é considerado um acontecimento humano de caráter complexo, universal e representando um grande problema de saúde pública em todo o mundo. Além de definir-se o suicídio como sendo a morte de uma pessoa causada por autoagressão, esse fenômeno ainda inclui desde motivação e ideação de autoextermínio, até o planejamento do ato, a tentativa e o óbito ${ }^{3}$. Nessa perspectiva, os dados apontam que cerca de um milhão de pessoas são acometidas por suicídio no mundo anualmente e a cada 45 segundos, uma pessoa em algum lugar do planeta se suicida. Países da Europa Oriental, América Central e América do Sul estão entre os que representam altos índices de suicídió.

Dados da Organização Mundial da
Saúde (OMS) de 2017, descreve em seu relatório sobre medidas preventivas de suicídio o Brasil sendo o oitavo pais com maior índice de suicídio. Aponta medidas passiveis de prevenção destacando medidas e estratégias mediante uma abordagem multisetorial de modo abrangente ${ }^{5}$.

Conceitualmente, a tentativa de suicídio compreende qualquer comportamento suicida não letal relacionado a um ato intencional contra a própria vida. Estudos revelam que o risco de suicídio aumenta proporcionalmente ao número de tentativas, as quais tendem a ser recorrentes, ressaltando que o histórico de tentativa de suicídio é o principal fator de risco para uma nova tentativa ${ }^{6,7}$.

O suicídio e a tentativa de suicídio são avaliados como um grande problema de saúde pública, o suicídio é um ato concreto, efetivado por uma pessoa que transparece consciência e noção da implicação final do seu ato. A expressão suicídio provém da forma latina "sui caedere" que expressa "matar-se"

As tentativas de suicídio são consideradas um evento complexo e multicausal, e a identificação de áreas de maior risco é uma ferramenta para o planejamento de estratégias mais eficazes para sua prevenção ${ }^{8}$.

De acordo com Vasconcelos Neto9, o comportamento suicida é intrínseco ao ser humano e representa um importante problema de saúde pública. O espectro desse comportamento envolve o suicídio (morte auto infligida), a tentativa de suicídio (TS) (comportamento autoagressivo sem evolução fatal) e a ideação suicida (pensar em acabar com a própria vida).

As tentativas de suicídio ocupam lugar importante no estudo das lesões autoprovocadas, uma vez que são um preditor importante para a morte autoinfligida ${ }^{10}$.

Estudos epidemiológicos são importantes. Conhecer o perfil das tentativas de suicídio permite não só identificar entre as vítimas quais estão sob maior risco como subsidiar as decisões voltadas a sua prevenção e ações para seu atendimento pelos profissionais de saúde e o profissional médico10. Portanto, este trabalho teve como objetivo analisar o perfil epidemiológico das tentativas de suicídio na população residente da cidade de São Paulo durante o ano de 2015.

\section{MÉTODOS}

Estudo transversal, descritivo, sobre notificações e internações decorrentes de tentativa de suicídio ocorridas na população residente da cidade de São Paulo, SP. $\mathrm{O}$ período da pesquisa compreende entre os meses de janeiro a dezembro de 2015 , pois os dados de 2015 foram os últimos disponibilizados no sítio eletrônico do Departamento de Informática do Sistema Único de Saúde (Datasus) no momento da análise. As fontes de dados são oriundas dos seguintes sistemas de informações de saúde: Sistema de Vigilância de Violência e Acidentes (SIVVA). 
Foram selecionadas para análise as notificações que, no campo da Ficha de Notificação Individual destinada ao registro de 'lesão autoprovocada', encontravam-se demarcadas como 'sim', e no campo de referência do 'autor da agressão', a 'própria pessoa. Para análise das notificações registradas no SIVVA/ Sinan, foram calculadas medidas de frequências segundo as seguintes variáveis:

- Sexo (feminino e masculino), Idade (em anos), Raça/cor da pele (branca; preta/parda; indígena; amarela); Local de ocorrência (residência; habitação coletiva; escola; via pública; e outros [local de prática esportiva, bar ou similar; comércio ou local de serviços; indústria; local de construção]); Meio utilizado (enforcamento; uso de objeto contundente; uso de objeto perfurocortante; uso de substância quente; envenenamento/intoxicação; uso de arma de fogo); Uso de álcool (sim; não);

- Evolução do caso: alta, internação, óbito no atendimento, transferência para serviço especializado. O estudo dispensou avaliação por parte de um Comitê de Ética em Pesquisa devido a utilização de dados secundários, não nominais, disponíveis para consulta pública no sitio eletrônico do Datasus 11, segundo resolução do Conselho Nacional de Saúde $-466 / 12$.

\section{RESULTADOS}

Foram identificadas 910 tentativas de suicídio na população residente na cidade de São Paulo, sendo 44, 5\% no sexo feminino e $55,5 \%$ no sexo masculino.

Os dados acerca a faixa etária da vítima: $3,2 \%$ crianças ( 0 a 9 anos de idade); 22,3\% adolescentes (10 a 19 anos de idade); $72,1 \%$ adultos (20 a 59 anos

\section{Tabela 1. Local da ocorrência das tentativas de suicídio da população residente} da cidade de São Paulo, 2015.

$\begin{array}{ccc}\text { Tipo de local da ocorrência } & \text { Notificações } & \mathbf{f ( \% )} \\ \text { Bar e afins } & 2 & 0,2 \\ \text { Escola } & 1 & 0,1 \\ \text { Local de trabalho } & 1 & 0,1 \\ \text { Residência } & 352 & 38,7 \\ \text { Via pública } & 21 & 2,3 \\ \text { Ignorado } & 533 & 58,6 \\ \text { Total } & 910 & 100,0\end{array}$

Tabela 2. Instrumento utilizado por tentativa de suicídio na população residente da cidade de São Paulo, segundo o sexo da vítima, 2015.

$\begin{array}{ccccc}\text { Instrumento utilizado } & \text { Feminino } & \mathbf{f ( \% )} & \text { Masculino } & \mathbf{f ( \% )} \\ \text { Afogamento } & 1 & 0,2 & 0 & 0,0 \\ \text { Arma Branca } & 28 & 6,9 & 44 & 8,7 \\ \text { Arma de Fogo } & 1 & 0,2 & 2 & 0,4 \\ \text { Enforcamento ou sufocação } & 6 & 1,5 & 12 & 2,4 \\ \text { Intoxicação / Envenenamento } & 322 & 79,5 & 388 & 76,8 \\ \text { Outros Meios } & 40 & 9,9 & 41 & 8,1 \\ \text { Precipitação de lugar elevado } & 7 & 1,7 & 18 & 3,6 \\ \text { Total } & 405 & 100,0 & 505 & 100,0 \\ \text { Fonte: autor, 2015. } & & & & \end{array}$

de idade) e $2,4 \%$ idosos ( 60 anos e mais de idade).

No que tange a variável raça/cor; branca $(36,7 \%)$, preto/pardo $(37,9 \%)$, indígena $(0,4 \%)$, amarela $(0,3 \%)$ e os demais dados foram ignorados.

Como mostrado na tabela1, o principal local de ocorrência é a própria residência da vítima $(38,7 \%)$.

Os dados da tabela 2. Mostram que o principal meio utilizado para as tentativas de suicídio é por intoxicação e envenenamento $(76,8 \%)$, em ambos os sexos.

Entre as vítimas por tentativa de suicídio $18,8 \%$ fizeram abuso de bebida alcoólica.

Os dados sobre a evolução do caso: $68,1 \%$ tiveram alta imediata, $14,0 \%$ internação, 10,3\% transferido para serviços especializados e $1,4 \%$ óbito no atendimento.

\section{DISCUSSÃO}

Durante o ano de 2015 foram notificados 910 casos de tentativas de suicídio na cidade de São Paulo. O cuidado com o fenômeno do suicídio deve vislumbrar, além de ações pontuais nos momentos de crises, ações educacionais, de saúde e sociais que tenham como norteadores o enlace do sujeito ao outro e à vida, o amparo do ser humano em seus momentos de sofrimento e um cuidado ético e integral com a promoção de vidas dignas. Pensar na integralidade como princípio básico para o cuidado de pessoas em risco para o suicídio, efetivada por meio da intersetorialidade e do cuidado em saúde pautado na relação sujeito-sujeito, deve ser o caminho para a construção de planejamentos que contribuam para a efetivação de políticas de valorização da vida. Deve-se romper a grande distância entre a teoria e práticas que ainda reforçam um modelo hospitalocêntrico, biologista e verticalizado na saúde, em prol de uma rede de cuidado em que todos os sujeitos sejam valorizados, com um trabalho articulado e dialógico entre gestão e atenção ${ }^{12}$.

De acordo com o presente estudo, as intoxicações e envenamento foram pre- 
dominantes em vítimas do sexo masculino; jovens e adultos de 20 a 49 anos. O suicídio e as tentativas de suicídio apresentam diferenças importantes entre homens e mulheres, uma vez que se adotam comportamentos autodestrutivos congruentes com as peculiaridades de cada gênero. Segundo Palma ${ }^{8}$, mundialmente os homens cometem suicídio de três a quatro vezes mais que as mulheres, resultado semelhante ao encontrado neste estudo para a cidade de São Paulo, onde os homens que tiveram maior número de tentativa de suicídio ${ }^{8}$.

Quanto ao local das tentativas de suicídios, houve predomínio na residência da vítima $(38,7 \%)$. Esse dado corrobora estudos no âmbito internacional e nacional, que referem a residência como ambiente predominante para concretização do suicídio, pelo livre acesso ${ }^{13}$.

No presente estudo apresentou as intoxicação e envenenamento foi predominante nas tentativas de suicídio, sendo o domicilio da vítima o principal local da ocorrência. Durante ao atendimento da vítima de tentativa de suicídio nos serviços de saúde, ressalta-se a importância da atuação multiprofissional, sendo um fator importante no que concerne ao cuidado do paciente, pois a troca de informações entre os profissionais pode ser eficaz na prevenção, diagnóstico, tratamento, notificação e acompanhamento das intoxicações ${ }^{14}$.

Diante do cenário das tentativas de suicídio por intoxicações, é relevante observar que apesar dos altos índices de ocorrência desses agravos na população, muitos casos são subnotificados. Almei$\mathrm{da}^{14}$, relata que a maioria das intoxicações é atendida e notificada em unidades de média a alta complexidade, indicando que a maioria consistiu em intoxicaçôes agudas e graves, sendo os quadros leves e crônicos não notificados ${ }^{14}$.

Como a tentativa de suicídio e o suicídio resulta de uma complexa interação de ordem psicológica, genética, cultural e socioambiental, abordagens multidisciplinares devem ser adotadas, respeitando-se as circunstâncias regionais. É necessária a obtenção contínua de dados, de maneira a se fortalecer em estratégias eficazes para a prevenção, formulação de políticas públicas e tratamento adequado das vítimas de tentativa de suicídio e se redirecionar em aquelas não efetivas ${ }^{15}$.

A variável raça/cor no presente estudo mostrou a tentativa de suicídio maior entre os pretos e pardo. Ao contrário do encontrado no presente estudo, as pessoas de raça/ cor branca são as que possuem maiores proporções de tentativa de suicídio no Brasil ${ }^{16}$.

Matos e Tourinho ${ }^{17}$, relatam que estudos associam raça a saúde mental, tendo a população negra maior tendência a esses transtornos, realidade encontrada no fato de suicídio figurar entre as 3 principais causas de morte na população parda em 2016. Uma possibilidade para justificar a maior concentração de negros nos Centro de Atenção Psicossocial (CAPS) em relação as Unidade Básicas de Saúde é a de que essa população esteja tendo menos acesso, afinal estudos revelam as diferenças no acesso e tratamento recebido nos serviços de saúde, bem como a existência de discriminação nos $\operatorname{mesmos}^{16,17}$.

Neste estudo foi encontrado que $18,8 \%$ das vítimas por tentativa de suicídio fizeram abuso de bebida alcoólica.

O consumo de bebidas alcoólicas é uma prática comum em todo mundo, causando frequentes e inúmeros problemas clínicos, psicológicos, profissionais e familiares. No Brasil, este problema acentua-se, pois, é vendida abertamente, sem que haja algum tipo de fiscalização, além das diversas propagandas de incentivo para o consumo nos diversos tipos de veículo de comunicação ${ }^{18}$.

Segundo Cordeiro ${ }^{18}$, a intoxicação alcoólica que antecede as tentativas de suicídios dos chamados alcoólatras deprimidos apresenta um ápice no consumo de álcool na véspera do evento. Trata-se de uma relação dose-resposta na qual quanto maior o consumo de álcool maior é a chance de comportamento suicida ${ }^{18}$.
Ressaltamos, a importância das informações adquiridas pela equipe multidisciplinar de saúde, melhor será o planejamento para uma abordagem terapêutica adequada, é importante salientar que cada indivíduo é diferente do outro e que o álcool atua de modo negativo no controle, na motivação, na atitude interna e contribui materialmente para o impulso do comportamento suicida ${ }^{18}$.

Nesse estudo identificamos como limitação uma possível subnotificação de casos, provavelmente devido a mudanças no fluxo de atendimento a urgências psiquiátricas. Diante de um fenômeno permeado por tabus e preconceito, em que grande parte dos profissionais de saúde não estão preparados adequadamente para atender essa demanda, é necessário $o$ investimento premente em formação continuada para profissionais de saúde nos diferentes serviços de saúde voltado para o atendimento a crise suicida ${ }^{19}$.

As subnotificações são ainda um entrave para a vigilância em saúde e para melhor identificação do comportamento suicida, entretanto, estas informações podem contribuir para que os profissionais de saúde sejam sensibilizados para a detecção oportuna deste comportamento e devida atenção ${ }^{20}$.

A maior parte dos atendimentos no setor saúde não demandaram os encaminhamentos necessários para suporte e acompanhamento adequado à vítima, nem mesmo para os casos de caráter repetitivo $^{20}$. Nesta casuística $10,3 \%$ foram encaminhados para serviços especializados.

Os hospitais, são instituições de grande abrangência social no território em que as vítimas estão inseridas, com a função principal de receber a maior demanda de casos graves, sendo eles de suicídio ou de diversos problemas, exercendo um papel notificador desses $\operatorname{casos}^{21}$.

Importante destacar, ainda, algumas das ferramentas estratégicas que os gestores e profissionais da saúde podem utilizar na tentativa de reduzir a ocorrência do agravo, objeto de análise neste estudo, são elas: promover a consciência de que o suicídio é um grande 
problema de saúde pública e que ele pode ser prevenido; desenvolver e ampliar a base de apoio para prevenção de suicídio; desenvolver e programar estratégias para minimizar os estigmas associados a ser usuário de saúde mental; e promover esforços para reduzir o acesso a meios e métodos letais de danos a si mesmo ${ }^{21}$.

\section{REFERÊNCIAS}

1. Tavares FL et al. Mortalidade por suicídio no Espírito Santo, Brasil: análise do período de 2012 a 2016. Av Enferm. 2020; 38 (1):66-76.

2. Ribeiro DB, Terra MG, Soccol KLS, Schneider JF, Camillo LA, Plein FAS. Motivos da tentativa de suicídio expressos por homens usuários de álcool e outras drogas. Rev Gaúcha Enferm. 2016 mar;37(1):e54896. doi: http://dx.doi. org/10.1590/19831447.2016.01.54896.

3. FélixT., OliveiraE., LopesM. V., ParenteJ. R., DiasM. S., \& MoreiraR. M. (2016). Fatores de risco para tentativa de suicídio: Produção de conhecimento no Brasil. Revista Contexto \& Saúde, 16(31), 173-185. https://doi.org/10.21527/21767114.2016.31.173-185.

4. Assumpção GLS, Oliveira LA, Souza MFS de. Depressão e suicídio: uma correlação. Rev. Pret. [Internet]. $7^{\circ}$ de março de 2018 [citado $10^{\circ}$ de novembro de 2020];3(5):312-33. Disponível em: http://periodicos.pucminas.br/index.php/pretextos/article/ view/15973.

5. Muller Alcantara Sônia, et al. Estratégias de prevenção e pósvenção do suicídio: Estudo com profissionais de um Centro de Atenção Psicossocial. Revista de Psicologia, IMED, 2017; 9 (2): 6 23. http://dx.doi.org/10.18256/2175-5027.2017.v9i2.1686.

6. Borba LO, Ferreira ACZ, Kalinke LP, Maftum MA, Maftum GJ. Fatores associados à tentativa de suicídio por pessoas com transtorno mental. Rev Min Enferm. 2020; 24: e-1284.

7. Pessoa DMS, Freitas RJM, Melo JAL, Barreto FA, Melo KCO, Dias ECS. Assistência de enfermagem na atenção primária à saúde de adolescentes com ideações suicidas. Rev Min Enferm. 2020; 24: e-1290.

8. Palma DCA, Santos ES, Ignotti E. Análise dos padrões espaciais e caracterização dos suicídios no Brasil entre 1990 e 2015. Cad. Saúde Pública 2020; 36(4): e00092819.

9. Vasconcelos Neto PJA, Moreira RS, Oliveira Junior FJM, Ludermir AB. Tentativa de suicídio, transtorno de estresse pós-traumático e fatores associados em mulheres do Recife. Rev Bras Epidemiol 2020; 23: E200010.

10. Bahia CA, Avanci JQ, Pinto LW, Minayo MCS. Notificações e internações por lesão autoprovocada em adolescentes no Brasil, 2007-2016. Epidemiol. Serv. Saude 2020; 29(2): e2019060,2020.
11. SIVVA - Sistema de Informação e Vigilância de Violências e Acidentes. [base de dados na internet]. São Paulo: Acidentes de Trânsito. [acesso em 23 de agosto de 2020]. Disponível em: http://www.prefeitura.sp.gov.br/ secretarias/saude.

12. Santos LA, Kind L. Integralidade, intersetorialidade e cuidado em saúde: caminhos para se enfrentar o suicídio Interface (Botucatu). 2020; 24: e190116.

13. Fernandes FY et al. Tendência de suicídio em adolescentes brasileiros entre 1997 e 2016. Epidemiol. Serv. Saude 2020; 29(4): e2020117.

14. Almeida ABM, Uchoa GF, Carvalho AMR. Epidemiologia das intoxicações medicamentosas registradas no sistema nacional de informações tóxico-farmacológicas de 2012-2016. Saúde e Pesqui. 2020; 13(2): 431-440.

15. Franck MC, Monteiro MG, Limberger RP. Mortalidade por suicídio no Rio Grande do Sul: uma análise transversal dos casos de 2017 e 2018. Epidemiol. Serv. Saude 2020, 29(2): e2019512.

16. Fernandes DAA, Ferreira NS, Castro JGD. Perfil epidemiológico das tentativas de suicídio em Palmas-Tocantins, de 2010 a 2014. Tempus, actas de saúde colet 2016, 10(4), 09-23.

17. Matos CCSA, Tourinho FSV. Saúde da População Negra: como nascem, vivem e morrem os indivíduos pretos e pardos em Florianópolis (SC). Rev Bras Med Fam Comunidade 2018; 13(40):113.

18. Cordeiro EL, Silva LSR, Mendes EWP, Silva LCL, Duarte VL, Lima ECMP. Tentativa de suicídio e fatores associados ao padrão uso e abuso do álcool. Rev. Eletrônica Saúde Mental Álcool Drog 2020; 16(1): 1-10.

19. Grigoletto AP, Souto VT, Terra MG, Tissot GL, Ferreira CN. Tentativas de suicídio notificadas em um hospital de ensino no estado do Rio Grande do Sul, 2014-2016. R. pesq.: cuid. fundam. online 2020; 12: 413-419.

20. Rodrigues MF, Oliveira PP, Silva HC, Pinheiro JMC. Comportamento suicida: o perfil epidemiológico das lesões autoprovocadas no estado de Goiás. Rev Cient Esc Estadual Saúde Pública Goiás "Cândido Santiago" 2020; 6 (2): e600003.

21. Santos Junior CJ, Santos IV, Silva JVS, Gomes VM, Ribeiro MC. Perfil de pacientes atendidos por tentativa de suicídio em um Hospital Geral de Emergências do estado de Alagoas, Brasil. Medicina (Ribeirão Preto. Online) 2019; 52 (3):223-230. 